\title{
Challenging PD-L1 expressing cytotoxic T cells as a predictor for response to immunotherapy in melanoma
}

\author{
Lieve Brochez $1,2,3,4$, Annabel Meireson 2,3 , Inès Chevolet ${ }^{2}$, Nora Sundahl ${ }^{3,5}$, Piet Ost ${ }^{3,4,5}$ \& Vibeke Kruse ${ }^{3,6}$
}

We read with great interest the report by Jacquelot $\mathrm{N}$ et al. ${ }^{1}$ on 'predictors of responses to immune checkpoint blockade in advanced melanoma'. Based on an ex vivo metastatic lymph node assay (stage III, $n=37$ ), the authors conclude that PD-L1 expression on circulating $\mathrm{CD}^{+}$and $\mathrm{CD}^{+} \mathrm{T}$ cells might be predictive biomarkers for resistance to CTLA-4 blockade. These observations were confirmed in vivo in another patient cohort, including 190 unresectable stage III and IV melanoma patients who were treated with ipilimumab.

We would like to report our observation that PD-L1 expressing cytotoxic $\mathrm{T}$ cells are associated with an altered immune climate and a negative impact on disease outcome in melanoma patients independent of treatment. It therefore meets the criteria of a prognostic biomarker ${ }^{2}$. Whether this biomarker is also predictive for response to immunotherapymeaning that the effect of immunotherapy is different in biomarker positive and negative patients-cannot be deduced from the retrospective single-arm (only treated patients) data in the study by Jacquelot et al. In fact this would require at least a twoarm design preferably in a randomized study, and known PD$\mathrm{L}^{+}$cytotoxic $\mathrm{T}$ levels in all patients. The only way to permit solid conclusions on its predictive value would be to demonstrate that the treatment-by-biomarker interaction is statistically significant, meaning that the levels of PD-L1 cytotoxic $\mathrm{T}$ cells are not only associated with worse disease outcome (prognostic), but are also associated with different response reactions to immunotherapy (predictive).

We investigated the in vivo immune profile in a cohort of 55 stage I to III patients having undergone only surgery. The median follow-up after diagnosis was 61 months (IQR: 38-112). During follow-up 15 patients $(27.3 \%)$ experienced disease relapse and six patients $(10.9 \%)$ died. Blood sampling for immune profiling was performed at a median time of 15 months (IQR: 4-60) after diagnosis. Patients' characteristics are presented in Supplementary Table 1.
The level of $\mathrm{PD}-\mathrm{L}^{+}{ }^{+} \mathrm{CD} 8^{+}$cells was strongly correlated with a number of markers indicating a negative immune climate (Table 1). In the current study cohort, a high frequency of PD-L1 $+\mathrm{CD}^{+}$cells was associated with decreased plasmacytoid dendritic cells ( $\mathrm{pDCs}$ ) and increased myeloid-derived suppressor cells (MDSCs). We previously demonstrated that these cell types are inversely correlated and have an independent prognostic effect on overall survival (OS) in melanoma patients ${ }^{3}$. Although PD-L1 ${ }^{+} \mathrm{CD}^{+}$cells were negatively correlated with $\mathrm{CD}^{+}$cells there was a strong correlation with Tregs (as detected by $\mathrm{CD} 3^{+} \mathrm{CD} 4^{+}$ $\mathrm{CD}_{25}{ }^{+}$FoxP3 $^{+}$expression) and the number of CTLA-4 expressing Tregs. On the other hand, levels of $\mathrm{PD}-\mathrm{L}^{+}{ }^{+} \mathrm{CD} 8^{+}$ cells were positively correlated with the serum kynurenine/tryptophan (kyn/trp) ratio, reflecting increased functional activity of indoleamine 2, 3-dioxygenase (IDO) in these patients. The intracellular enzyme IDO degrades tryptophan to kynurenine which is considered to be a mechanism attributing to immune tolerance. IDO has been demonstrated to be a negative prognostic marker in a variety of cancers ${ }^{4}$. The above results imply that PDL1 expression on cytotoxic T-cells is in close interplay with other immune-inhibitory cell types and molecules, all together contributing to the installation of a negative immune climate.

The clinical follow-up of the included patients is illustrated in Fig. 1a. Patients who died from melanoma $(n=6$; median time of 9.5 months after blood sampling, IQR: 3.8-28.8) had higher levels of $\mathrm{PD}-\mathrm{L1}^{+} \mathrm{CD}^{+}$cells $(p=0.039$, Mann-Whitney $U$-test). For survival analysis, the percentage of circulating $\mathrm{PD}-\mathrm{L} 1^{+} \mathrm{CD}{ }^{+}$ cells was dichotomized by ROC analysis into "low" $(<0.1505 \%$ of live PBMCs) and "high" ( $>0.1505 \%$ of live PBMCs). High levels of $\mathrm{PD}-\mathrm{L}^{+}{ }^{+} \mathrm{CD} 8^{+}$cells were observed to be a negative prognostic marker for OS in univariate analysis $(p=0.038$, log-rank test, Fig. 1b). Multivariate analysis with a Cox proportional hazard regression model including disease stage (local versus regional disease) demonstrated $\mathrm{PD}-\mathrm{L} 1{ }^{+} \mathrm{CD} 8{ }^{+}$cells to be an independent negative prognostic marker on OS $(p=0.047$; HR: $7.42,95 \% \mathrm{CI}$ :

\footnotetext{
${ }^{1}$ Department of Dermatology, Ghent University Hospital, Corneel Heymanslaan 10, 9000 Ghent, Belgium. ${ }^{2}$ Dermatology Research Unit, Ghent University Hospital, Corneel Heymanslaan 10, 9000 Ghent, Belgium. ${ }^{3}$ Immuno-Oncology Network Ghent (ION Ghent), Ghent, Belgium. ${ }^{4}$ Cancer Research Institute Ghent (CRIG), Ghent, Belgium. ${ }^{5}$ Department of Radiotherapy, Ghent University Hospital, Corneel Heymanslaan 10, 9000 Ghent, Belgium. ${ }^{6}$ Department of Medical Oncology, Ghent University Hospital, Corneel Heymanslaan 10, 9000 Ghent, Belgium. These authors contributed equally: Lieve Brochez,

Annabel Meireson. Correspondence and requests for materials should be addressed to L.B. (email: lieve.brochez@ugent.be)
} 
1.03-53.67). Moreover, the frequency of PD- $\mathrm{L}^{+}{ }^{+} \mathrm{CD} 8^{+}$cells was increased in patients who were close to disease relapse or disease related death. The shorter the time frame between blood sampling and relapse or death, the higher the frequency of $\mathrm{PD}-\mathrm{L1}^{+} \mathrm{CD} 8^{+}$ cells (resp. $p=0.005, \mathrm{CC}-0.975$ and $p=0.005, \mathrm{CC}-0.943$ ).

Jacquelot et al. state that prediction of patients' response to immunotherapy by dynamic biomarkers represent an unmet medical need. More important than finding predictors for immunotherapy (no-) response may be the development of tools to routinely monitor the immune response before and during immunotherapy. If negative immunological signs are observed, there might be an opportunity for early intervention to try to reverse this climate. In 10 stage IV melanoma patients treated with ipilimumab and stereotactic body radiotherapy, nonresponders showed an increase in the kyn/trp ratio during treatment compared to baseline ${ }^{5}$. Wang et al. ${ }^{6}$ recently demonstrated that increasing kyn/trp ratio after chemoradiation predicts worse survival outcome in lung cancer patients. As the kyn/trp ratio is reflecting IDO activity, these patients subgroups might be candidates for an IDO-inhibitor.

Immunomonitoring before and during cancer (immuno-) therapy, and sequential therapy adapted to the observationsrather than upfront combinations-may be a new step in the era of personalized immunotherapy.

\section{Methods}

Patients. Fifty-five patients with melanoma were included in this study, with a median follow-up of 61 months (38.0-112.0) after diagnosis and 46 months

\section{Table 1 Association of PD-L1 ${ }^{+}$CD8 $^{+}$cells with other systemic immune markers}

\begin{tabular}{|c|c|c|}
\hline & Spearman correlation coefficient & $P$-value \\
\hline \multicolumn{3}{|l|}{ Serum } \\
\hline $\begin{array}{l}\text { Kyn/Trp } \\
\text { PBMCs: T-lymphocytes }\end{array}$ & PBMCs: T-lymphocytes & 0.014 \\
\hline $\mathrm{CD}^{+}$ & 0.367 & 0.006 \\
\hline $\mathrm{CD}^{+}{ }^{+}$ & -0.446 & 0.001 \\
\hline Tregs & 0.414 & 0.002 \\
\hline CTLA-4 ${ }^{+}$Tregs & 0.399 & 0.003 \\
\hline $\mathrm{CD}^{+}$ & -0.589 & $<0.001$ \\
\hline \multicolumn{3}{|l|}{ PBMCs: dendritic cells } \\
\hline $\mathrm{MDSC} / \mathrm{pDC}$ & 0.304 & 0.024 \\
\hline
\end{tabular}

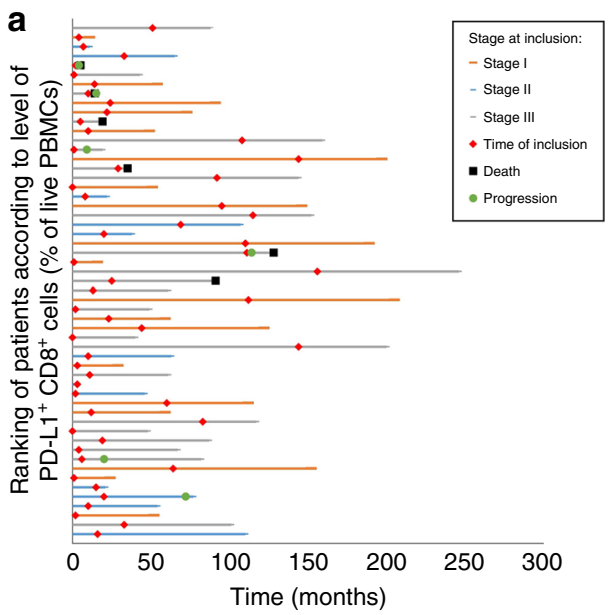

(22.0-55.0) after inclusion (i.e., the time of blood collection). Only patients who did not receive systemic therapy were included, to avoid any possible influence on marker expression and survival analysis. Detailed patient characteristics can be found in Supplementary Table 1 . This study was approved by the ethical committee of Ghent University Hospital. All included patients provided informed consent before enrollment in the study.

PBMC isolation. Peripheral blood mononuclear cells (PBMCs) were isolated from heparinized venous blood by centrifugation on a Ficoll-Hypaque gradient (GE Healthcare, Uppsala, Sweden) within a $4 \mathrm{~h}$ of venipuncture. The cells were washed three times and cryopreserved at $-80^{\circ} \mathrm{C}$ in fetal bovine serum (FBS, Invitrogen, Merelbeke, Gelbium) supplemented with 10\% dimethyl sulfoxide (DMSO, Merck, Darmstadt, Germany) and 1\% penicillin-streptavidine (Invitrogen, Merelbeke, Belgium) until analysis. Cells were thawed by submersion at $37^{\circ}$ for $1-2 \mathrm{~min}$ and resuspended in a medium containing Iscove's Modified Dulbeco's Medium (IMDM) supplemented with $20 \%$ FBS and $1 \%$ glutamine.

Flow cytometry. MDSCs were characterized by the HLA-DR ${ }^{-}$lineage ${ }^{-}(\mathrm{CD} 3$, $\mathrm{CD} 19$, and CD56) $\mathrm{CD}_{3}{ }^{+} \mathrm{CD} 1 \mathrm{~b}^{+}$phenotype. Dendritic cells were characterized by the HLA-DR ${ }^{+}$lineage ${ }^{-}(\mathrm{CD} 3, \mathrm{CD} 14, \mathrm{CD} 16, \mathrm{CD} 19, \mathrm{CD} 20$, and CD56) phenotype, pDCs are $\mathrm{CD} 123^{+} \mathrm{CD} 11 \mathrm{c}^{-}$. Tregs were defined as $\mathrm{CD} 3^{+} \mathrm{CD} 4^{+} \mathrm{CD} 25^{+}$ FoxP3 $^{+}$and cytotoxic T-cells as $\mathrm{CD}^{+} \mathrm{CD}^{+}$cells. All antibodies used in this study were fluorescently conjugated mouse anti-human monoclonal antibodies. The following antibodies were purchased from BD Biosciences; CD3 BV421 (563797), CD4 APC-Cy7 (561839), CD25 FITC (560990), CD33 BV421 (562854), CD11b APC-Cy7 (560914), CD123 BV421 (562517). The following antibodies were purchased from eBioscience; B7-H1 PE-Cy7 (25-5983-42), CD8 APC (90170087-025), CD3 FITC (11-0038-41), CD19 FITC (11-0199-41), CD56 FITC (110569-41), CD14 APC (17-0149-41), CD11c APC (17-0116-41), HLA-DR PerCPCy5.5 (45-9956-42). For intracellular stainings, after surface staining PBMCs were fixed and permeabilized with fixation/permeabilization solution (BD Biosciences), and then stained with CTLA-4 APC (BD Biosciences, 560938) and FoxP3 PerCPCy5.5 (eBioscience, 45-4776-42) antibodies. Live/dead staining was performed using Live/dead ${ }^{\circledast}$ fixable aqua dead cell stain (Life Technologies Europe). Cells were analyzed on a FACSCanto ${ }^{\text {sx }}$ II flow cytometer (BD Bioscience, Erembodegem, Belgium) using FlowJo software (Tree Star Inc, Ashland, OR, USA). For setting the gates, isotype, and fluorescence-minus-one (FMO) controls were used. To provide a representative sample a median amount of 500,000 cells was analysed per cell type ( $\min 261,000-\max 569,750$ ). The reported frequencies of circulating cell types are percentages of live PBMCs, except for Treg frequencies which are percentages of $\mathrm{CD} 4^{+}$cells.

Ultra-performance liquid chromatography (UPLC). UPLC-MS/MS (Waters Acquity TQD) was conducted to quantify tryptophan and kynurenin on frozen patient sera, according to previously published methods ${ }^{7}$.

Statistical analysis. All statistical analyses were performed using SPSS 24.0 (SPSS Inc, Chicago, IL, USA), a $P$-value less than 0.05 was considered statistically significant (double-sided). Shapiro-Wilk test demonstrated that the data had no normal distribution and therefore non-parametric statistical tests were used for further analysis. Spearman correlation coefficients (CC) were calculated to evaluate correlations between continuous variables.. Values between two groups were compared by a two-tailed Mann-Whitney $U$-test. OS was estimated by the

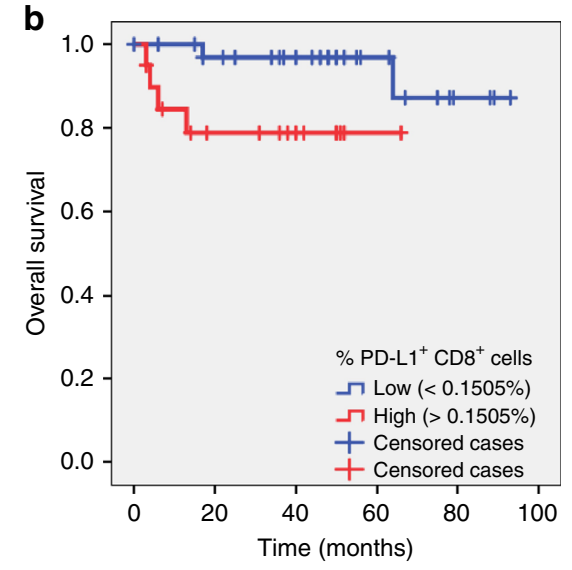

Fig. 1 PD-L1+ ${ }^{+} \mathrm{CD} 8^{+}$level according to disease stage and overall survival. Swimmer plot showing the relation between the ranking of PD-L $1^{+} \mathrm{CD} 8^{+}$cells (according to frequency) and clinical follow-up. b Kaplan-Meier curves of overall survival according to the levels of PD-L1+ CD $8^{+}$cells (\% of live peripheral blood mononuclear cells) 
Kaplan-Meier method and compared by the log-rank test. OS was defined as the time from the date of blood collection to death. Multivariate survival analysis was performed utilizing the Cox proportional hazard model. Dichotomization of the level of PD-L1 ${ }^{+} \mathrm{CD}{ }^{+}$cells was performed by means of ROC analysis maximizing Youden's statistics using $\mathrm{R}$ software. A value of $0.1505 \%$ was set as a cut-off (sensitivity $91.7 \%$, specificity $48.3 \%$ ).

Data availability. The dataset generated during and/or analysed for the current study are available from the corresponding author on reasonable request.

Received: 23 December 2017 Accepted: 13 June 2018

Published online: 26 July 2018

\section{References}

1. Jacquelot, N. et al. Predictors of responses to immune checkpoint blockade in advanced melanoma. Nat. Commun. 8, 592 (2017).

2. Ballman, K. V. Biomarker: predictive or prognostic? J. Clin. Oncol. 33, 3968-3971 (2015).

3. Chevolet, I. et al. Clinical significance of plasmacytoid dendritic cells and myeloid-derived suppressor cells in melanoma. J. Transl. Med. 13, 9 (2015).

4. Brochez, L., Chevolet, I. \& Kruse, V. The rationale of indoleamine 2,3dioxygenase inhibition for cancer therapy. Eur. J. Cancer Oxf. Engl. 199076 , 167-182 (2017).

5. Sundahl, N. et al. A phase 1 dose escalation trial of ipilimumab and stereotactic body radiotherapy in metastatic melanoma. Int. J. Radiat. Oncol. Biol. Phys. 100, 906-915 (2017).

6. Wang, W. et al. IDO immune status after chemoradiation may predict survival in lung cancer patients. Cancer Res. 78, 809-816 (2017).

7. Schefold, J. C. et al. Increased indoleamine 2,3-dioxygenase (IDO) activity and elevated serum levels of tryptophan catabolites in patients with chronic kidney disease: a possible link between chronic inflammation and uraemic symptoms. Nephrol. Dial. Transplant. 24, 1901-1908 (2009).

\section{Author contributions}

L.B., V.K., N.S., and P.O. provided patient samples and clinical information. I.C. and A.M. conducted experiments. A.M. and L.B. performed statistical evaluations and prepared the figures. The correspondence was written by L.B. and A.M. with contributions from P.O., N.S., and V.K.

\section{Additional information}

Supplementary Information accompanies this paper at https://doi.org/10.1038/s41467018-05047-1.

Competing interests: L.B. was participant in advisory board Incyte, München, Germany, June 2017 and gave an internal training for Incyte European division, Amsterdam, October 2017. The remaining authors declare no competing interests.

Reprints and permission information is available online at http://npg.nature.com/ reprintsandpermissions/

Publisher's note: Springer Nature remains neutral with regard to jurisdictional claims in published maps and institutional affiliations.

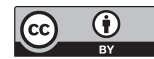

Open Access This article is licensed under a Creative Commons Attribution 4.0 International License, which permits use, sharing, adaptation, distribution and reproduction in any medium or format, as long as you give appropriate credit to the original author(s) and the source, provide a link to the Creative Commons license, and indicate if changes were made. The images or other third party material in this article are included in the article's Creative Commons license, unless indicated otherwise in a credit line to the material. If material is not included in the article's Creative Commons license and your intended use is not permitted by statutory regulation or exceeds the permitted use, you will need to obtain permission directly from the copyright holder. To view a copy of this license, visit http://creativecommons.org/ licenses/by/4.0/.

(C) The Author(s) 2018 